\title{
SenseCam intervention based on Cognitive Stimulation Therapy framework for Early-Stage Dementia.
}

\author{
Paulina Piasek $^{*}$, Kate Irving ${ }^{*}$ and Alan F. Smeaton ${ }^{+}$ \\ ${ }^{*}$ School of Nursing and ${ }^{+}$CLARITY: Centre for Sensor Web Technologies \\ Dublin City University \\ Dublin, Ireland
}

\begin{abstract}
Estimates suggest that Ireland will have 50,000 people with dementia by 2016 and over 100,000 by 2036 . Combined with European total costs estimated in 2005 at $€ 130$ billion. In the absence of a cure for dementia there is a real need to develop pervasive, user-centered technologies to enhance the well-being and quality of life of people with dementia. This study explores the use of Microsoft SenseCam images within the principles of Cognitive Stimulation Therapy (CST) to engage people with early stage dementia in meaningful discussion. The SenseCam intervention, like the CST approach, is aimed at general enhancement of quality of life and global cognitive and social functioning.
\end{abstract}

Keywords: Early stage dementia; SenseCam; Cognitive stimulation therapy; Technology

\section{INTRODUCTION}

The purpose of this research is to contribute to the knowledge of meaningful interventions in early stage dementia care. It will explore the discussion encouraged by viewing images derived from Microsoft SenseCam, a wearable camera, within the principles of Cognitive Stimulation Therapy (CST).

CST focuses on strengths and abilities and is careful to avoid situations which erode self-esteem [1]. CST as an approach relies heavily on images to generate discussion. In this instance the images derived from SenseCam are personal to the individual. SenseCam is a lightweight wearable digital camera which passively takes images of the wearers' activities throughout the day. It does not have a viewfinder or a display that can be used to frame photos; instead SenseCam is fitted with a wide-angle (fish-eye) lens that maximizes its field-ofview. This ensures that nearly everything in the wearer's view is captured by the camera. SenseCam also contains a number of electronic sensors, including light-intensity, a passive infrared (body heat) detector, accelermoeters, and a temperature sensor, which are used together to automatically trigger a photograph to be taken [2]. SenseCam takes about 3,000 images in a typical day.

In this project, the focus of the intervention is creating meaningful discussion with the person with dementia. Because remembering is an act of communication, re-telling past experiences is integral to social interaction and creates interpersonal bonds. A broad range of measures and qualitative data will be collected to evaluate the extent to which the images from an individuals own life generate discussion, and such discussions effect quality of life, communication skills and carers' strain for example.

\section{THE SCALE OF DEMENTIA}

There are 37,000 people with dementia in Ireland [3]. By the year 2026 it is estimated this number will increase to 52,000 [3]. In the European Union approximately 5.1 million people live with dementia and within the next 50 years this number will raise to approximately 11.9 million dementia cases [4]. Based on several meta-analysis of epidemiological studies, [5] calculated 8.5 million dementia sufferers in the year 2010 and the number will exceed 16.2 million in the year 2050, in the whole of Europe including eastern sub-regions. In comparison the estimated number of people with dementia in Australia is 200,000 and will exceed 730,000 by the year 2050 [6]. The current research on prevalence rates in dementia predicts dementia cases per year will increase regardless of geographical location [3-5]. Contrarily, the working-age population will decrease during the next 50 years [5].

Dementia is a serious loss of cognitive ability beyond that which might be expected from normal aging which affects social activities, relationships or work. The above criteria set out in the Diagnostic and Statistical Manual of Mental Disorders is most commonly used for diagnosis of the disease [7]. Dementia is an umbrella term for many different diseases, all of which have similar symptoms, but different aetiologies. The onset of dementia is generally insidious and there is no definitive biological marker of disease onset. Because of this the diagnosis of dementia is a complex process [8]. The scale for staging of dementia enables approximate classification of the disease, into mild, moderate or severe, with the latter presenting high cognitive impairment and the former very low cognitive impairment [9]. The staging of the disease allows engaging the person with dementia with appropriate interventions, therapies and care especially in the early stages of the disease [10].

There is growing evidence that early identification of dementia has the potential to be beneficial to sufferers and their families and so there is increasing medical and public consensus that diagnosis should be made as early in the illness 
as possible [11]. There are many challenges after dementia diagnosis is issued to both the person and their family which include communication and social withdrawal directly affecting the well-being of the person with dementia [12]. One study found benefits from early-targeted psychosocial intervention for people with dementia and their carers [13]. The results indicated the possibility of improving memory performance and maintaining carer mood through use of targeted psychosocial intervention in the early stages [13].

In order to deliver a high standard of care within the financial constraints likely to face health services over the next decade it is essential to develop our knowledge of strategies that can improve independence and quality of life for people with dementia. As currently there is no cure for dementia and early diagnosis and intervention are the expressed aim of every public policy on the disease, a wider range of legitimate supportive options are necessary. A recent study identified the need for pervasive technologies to move from managing illness to maintaining well-being [14]. Many of these technologies are being developed to enhance the well-being and quality of life of people with dementia. Including this research on SenseCam therapy for people with early stage dementia.

\section{TECHNOLOGY AND DEMENTIA}

Technology has the potential to promote independence and enhance quality of life of the person with dementia. Pervasive health care technologies are promising and can make very significant difference to the lives of people with dementia and their carers [15]. The benefits of using technology in dementia care has been demonstrated through recent initiatives including ASTRID [16] and ENABLE (Enabling technologies for people with dementia) [17]. These projects not only describe the opportunities innovative technology can create, but also the technological solutions to common challenges and the ethical considerations associated with them $[16,17]$.

Research has demonstrated that there is a wide variety of technologies available to both people with dementia and their carers. They can be divided into 4 main categories, namely (a) Monitoring and Safety (b) Communication and Interaction (c) Supporting daily life Independence and (d) Memory training. The following are examples of research within these categories.

\section{A. Monitoring and Safety.}

Monitoring and safety technologies used in dementia care include GPS systems which enable tracking of the person with dementia and alerting the carer if safe boundaries are crossed. Bed Occupancy Sensors are also popular in care settings, allowing monitoring patients by nursing staff [18].

\section{B. Communication and Interaction.}

The CIRCA project includes a touch screen computer with variety of media including generic photographs, music and video. It aims at increasing communication and relationship between the person with dementia and the caregiver through technologically innovative reminiscence [19].

\section{Supporting daily - life Independence.}

The ENABLE multi-national project assessed the effectiveness of devices such as calendars, picture telephones, item locators, automated night lamps, and gas cooker monitors among people with dementia. The results for Ireland indicated that devices such as telephones and calendars were most successful because of their ease of use and low technical difficulty [15].

\section{Memory Training.}

GRADIOR is a multimedia system designed for neuropsychological rehabilitation and cognitive assessment. Training focuses on the least-deteriorated cognitive functions and content for training is chosen by the carer or therapist [20].

The safety and security of individuals with dementia still dominates the technological innovations. Less research is carried out on how to enhance quality of life and general wellbeing. Many of the monitoring technologies can be seen as supporting the carer rather than the person with dementia. According to [21] quality of life is more than just feeling safe and secure; there is a real need to develop more innovative technologies aimed at improving the quality of life of the person with dementia.

Essentially, our research addresses the communication and interaction technology category from the above. However it also supports independence in that if successful in enabling a person with dementia to maintain their conversational skills it will consequently help address social isolation. SenseCam images have also been found to improve memory in patients with memory impairments [22], hence this technology has potential as a memory training technology although this is not the central focus of this study.

\section{THERAPEUTIC APPROACH}

Our research uses an innovative approach by combining SenseCam images with principles of CST to encourage discussion and improve global cognition. Aspects of CST to be adopted include a framework of 14 sessions of themed activities run over a seven week period. The sessions have some basis in reminiscence, where meaningful discussion about memories from one's life is the key to interaction, enjoyment, increased contribution and engagement [23]. CST aims to actively stimulate and engage people with dementia, whilst providing an optimal learning environment.

\section{RESEARCH OBJECTIVES}

Our research explores the response of participants to viewing images derived from Microsoft SenseCam. A secondary aims includes exploring whether the images enable rich opinion based discussion, under what conditions, and whether discussing such images is meaningful and enjoyable to individuals. The findings will contribute to the knowledge of meaningful interventions in the Early-Stage Dementia care. This research will be conducted over a 36 month period in an exploratory and descriptive approach using the Multiple Case Study method [24]. Three individual case studies will be used to collect in-depth data within their context about a complex issue.

Three individuals with early stage dementia who have a carer will be recruited. Participants will be asked to wear SenseCam while they go about their everyday life, for the duration of seven weeks. Participants will decide with the help of their carers when they wish to wear the SenseCam and under what social conditions (e.g. alone at home, leisure time 
activities, socializing with others, etc.). Participants will also be made aware of a privacy button on the camera allowing them to switch the SenseCam off at any time they wish. For the period of these seven weeks the therapist and the researcher will call at pre-arranged appointments twice a week, 45 minutes each. During this time the therapist will view the images and engage the participant in discussions about the images using software which automatically structures the thousands of SenseCam images captured each day, into "events". The event-based browsing software developed in the CLARITY centre [25] allows huge amounts of SenseCam data to be navigated easily. The participant, the carer and the researcher will keep a journal each reflecting on observations throughout all sessions during the seven week period. The researcher will observe the therapist and participant engaging in CST, noting reflections in the journal regarding the process of administration, participant enjoyment and any other comments. A tape recorded interview including responses to standardized measures, medical and social history will be undertaken before and after the seven week therapy course.

A variety of qualitative and quantitative data will be used to ensure the experience of discussion encouraged through viewing the images derived from SenseCam is explored through multiple lenses. This will allow multiple facets of the phenomenon to be revealed. This is an original interpretation of cognitive stimulation and thus needs careful study in order to explore the possibilities of its use and its effects.

Qualitative data will include medical and social history, a tape recorded interview with the carer and the person with dementia before and after the course of the therapy and journals kept by the researcher, the carer and the person with dementia reflecting on observations throughout the sessions from the perspective of the author. Quantitative data will include responses to standardized measures collected before and after the therapy course. There is evidence that questionnaire responses are important factors to take into consideration in evaluating any therapeutic intervention [10]. The use of these questionnaires will also allow some preliminary comparison of the effect sizes of previous CST research although it must be stressed that any such comparisons will be tentative. Nonparametric tests of repeated measures will be used on the quantitative data. Once the quantitative data is analyzed, results will be placed in arrays with the qualitative data. Arrays can be grouped by source, chronologically, by proposition question or thematically. Typically the data is combined and recombined in several different ways in order to get a thorough understanding of the nuances and the different point of view in each case. As the proposition questions are addressed information can be triangulated.

\section{Challenges, Risks AND Benefits}

This study will use images from the participants' everyday life for the purpose of the therapy. During which the therapist will view the images and engage the participant in discussions about them. Using past memories is usually an enjoyable experience like tapping into a strength many people with dementia have. Furthermore, the study is low risk as the CST sets clear ground rules that the focus is not on remembering specific facts, events or people, personal difficulties or traumatic incidents. The focus is on pleasurable therapeutic process, stimulating rich opinion rather than fact based discussion. The opinions may be amusing, sad, and unusual but they will never be wrong. Nevertheless, some participants may have unhappy (even traumatic) memories which could be triggered by viewing some of the images. Participants may find it challenging viewing images of their life that they may not remember. Also they may draw on their life memories or may have view their images negatively, regretfully, as an embarrassment, or for unanticipated reasons may become distressed during the therapy.

The research team acknowledges this issue and has set up safeguards to deal with such situations and to minimize the risk to the participants. In the event that this occurs participants will never be pushed into exposing painful memories against their will. If however the participant necessitates talking further about it, the therapist will enable them to discuss their distress and will be helped reflect on the best course of immediate action, including taking a break or discontinuing the therapy.

Currently, people with dementia and their families in Ireland rarely receive any intervention in the early stages of the disease; participation in this study will provide free intervention for both the person with the dementia and their carer. It is possible that there will be direct benefits to the person with dementia and the carer in terms of improved cognitive or psychosocial well-being. Indirectly this will contribute to the currently small literature base on meaningful responses to dementia.

\section{REFERENCES}

[1] A. Spector, et al. (2003 09). Efficacy of an evidence-based cognitive stimulation therapy programme for people with dementia. British Journal of Psychiatry 183(3), pp. 248-254.

[2] Microsoft Corporation. Using SenseCam to Alleviate Memory Loss [Online]. http://research.microsoft.com/enus/um/cambridge/projects/sensecam/memory.htm [accessed: 01/11]

[3] E. O'Shea and S. O'Reilly. (2000 03). The economic and social cost of dementia in ireland. Int. J. Geriatr. Psychiatry 15(3), pp. 208-218.

[4] C. Berr, J. Wancata and K. Ritchie. (2005 08). Prevalence of dementia in the elderly in europe. European

Neuropsychopharmacology 15(4), pp. 463-471.

[5] J. Wancata, et al. (2003 10). Number of dementia sufferers in europe between the years 2000 and 2050. European Psychiatry 18(6), pp. 306-313.

[6] K. J. Anstey, et al. (2010 21). Estimates of probable dementia prevalence from population-based surveys compared with dementia prevalence estimates based on meta-analyses. BMC Neurology 10 
[7] American Psychiatric Association., Diagnostic and Statistical Manual of Mental Disorders IV, Washington, DC: American Psychiatric Association, 1995,

[8] H. Chui, "Diagnosis," in Evidence-Based Dementia Practice N. Qizilbash, L. S. Schneider, H. Chui, P. Tariot, H. Brodaty, J. Kaye and T. Erkinjuntti, Eds. Oxford: Blackwell Science Ltd, 2003,

[9] C. P. Hughes. (1982 06). A new clinical scale for the staging of dementia. British Journal of Psychiatry 140pp. 566572 .

[10] E. Moniz-Cook and J. Manthorpe, Early Psychosocial Interventions in Dementia: Evidence-Based Practice. London: Jessica Kingsley Publishers, 2009,

[11] National Audit Office, "Improving services and support for people with dementia." The Stationary Office, London, 2007.

[12] H. J. Husband, "What do we tell people with dementia about their diagnosis and how do we tell them," in Early Psychosocial Interventions in Dementia: Evidence-Based Practice E. Moniz-Cook and J. Manthorpe, Eds. Jessica Kingsley Publishers, 2009,

[13] E. Moniz-Cook, et al. (1998 08). A preliminary study of the effects of early intervention with people with dementia and their families in a memory clinic. Aging \& Mental Health 2(3), pp. 199-211.

[14] B. Arnrich, et al. (2010 03). Pervasive healthcare: Paving the way for a pervasive, user-centered and preventive healthcare model. Methods Inf. Med. 49(1), pp. 67-73.

[15] I. Hagen, et al. (2007 05). "It gives me a sense of independence" - findings from Ireland on the use and usefulness of assistive technology for people with dementia. TECHNOL DISABIL 19(2-3), pp. 133-142.

[16] Anonymous (2000 2000). ASTRID: Introducing assistive technology... A social and technological response to meeting the needs of individuals with dementia. J DEMENT CARE 8(4), pp. 18-19.
[17] Anonymous (2007 05). Special issue: Technology in dementia care. TECHNOL DISABIL 19(2-3), pp. 53-152.

[18] W. Carswell, et al. (2009 07). A review of the role of assistive technology for people with dementia in the hours of darkness. Technol. Health Care 17(4), pp. 281-304.

[19] A. J. Astell, et al. (2010 07). Using a touch screen computer to support relationships between people with dementia and caregivers. Interact Comput 22(4), pp. 267-275.

[20] M. Franco, K. Jones, B. Woods and P. Gomez, "GRADIOR: A personalised computer-based cognitive training programme for early intervention in dementia." in Early Psychosocial Interventions in Dementia: EvidenceBased Practice.E. Moniz-Cook and J. Manthorpe, Eds. Jessica Kingsley Publishers, 2009,

[21] I. Hagen, et al. (2007 05). Designing technology to support quality of life of people with dementia. TECHNOL DISABIL 19(2-3), pp. 103-112.

[22] E. Berry, et al. (2007 08). The use of a wearable camera, SenseCam, as a pictorial diary to improve autobiographical memory in patient with limbic encephalitis: A preliminary report. Neuropsychological Rehabilitation 17(4-5), pp. 582601.

[23] D. Brooker, "Therapeutic activity," in A Handbook of Dementia Care C. Cantley, Ed. Buckingham: Open University Press, 2001,

[24] R. K. Yin., Case Study Research: Design and Methods, ,4th ed., vol. 5, Thousand Oaks, Calif.: Sage Publications, 2009 ,

[25] A.R. Doherty and A.F. Smeaton, "Automatically Segmenting LifeLog Data into Events," WIAMIS, pp.20-23, 2008 Ninth International Workshop on Image Analysis for Multimedia Interactive Services, 2008. 